\title{
Aplicación \\ basada \\ en \\ la \\ Metodología interactivo \\ STEAM: Un juego
}

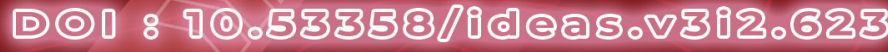

\author{
John Wladimir Simisterra Quiñónez ${ }^{1}$, Darío Fernando Yépez Ponce ${ }^{1,2}[0000-0002-2311-9368]$ y Héctor \\ Mauricio Yépez Ponce ${ }^{20000-0003-0625-1065]}$ \\ ${ }^{1}$ Instituto Superior Tecnológico Luis Tello, 080101 Esmeraldas, Ecuador \\ ${ }^{2}$ Ardutech.ec, 100450 Otavalo, Ecuador
}

jwsimisterra@istluistello.edu.ec,dfyepez@istluistello.edu.ecymauroyepez32@gmail.com

\section{RESUMEN}

Resumen. Las aplicaciones basadas en software de computadoras han sido uno de los avances más significativos para la humanidad, las mismas han cambiado la vida del hombre y el control de los procesos. En el ámbito educativo, las TIC han transformado los procesos de enseñanzaaprendizaje obligando a estudiantes y docentes a mejorar sus competencias digitales. En este contexto, se ha desarrollado un juego interactivo basado en la metodología STEAM para los niños y niñas del primer año de educación básica. La aplicación fomenta en los educandos el razonamiento lógico, matemático y espacial mientras juegan; las instrucciones ingresadas son simuladas en el software por medio de animaciones y si no hay errores se habilita un botón que permite enviar las ordenes programadas por Wi-Fi a un robot móvil. El juego implementado tiene una interfaz interactiva, fomenta las bases de la programación y le permite al educando aprender mientras juega.

Palabras Clave: Aplicación STEAM, Juego STEAM, Metodología STEAM, Robótica educativa. 


\section{Introducción}

La utilización del software educativo en los procesos de enseñanza-aprendizaje se inició hace varios años [1]. En [2], evidencian que una correcta selección de la tecnología contribuye en la mejora de la participación de los educandos y en la consecución de mejores resultados en el aprendizaje. El uso de las aplicaciones en los procesos de enseñanza-aprendizaje, es un método que se ha proliferado en los últimos años debido a que permiten comunicarse, relacionarse, aprender y enseñar dentro y fuera de clase [3].

De acuerdo con [4], la metodología STEAM (Science, Technology, Engineering, Art and Mathematics) ha contribuido en la popularización de la ciencia y la tecnología en la educación básica y la consolidación de competencias científicas en los países desarrollados. En [5], [6] manifiestan que el mundo de hoy requiere de docentes con una formación STEAM para que integre las ciencias, matemática, ingeniería, robótica, artes, la creatividad y las TIC en la consolidación de los aprendizajes desde una perspectiva transdisciplinar haciendo la ciencia más atractiva y desarrollando el pensamiento crítico, científico y tecnológico de los educandos.

El sistema pedagógico se halla en plena transformación y en este cambio, la robótica educativa ha surgido también como herramienta de aprendizaje colaborativo que combina del hardware y software para que el educador imparta eficientemente el conocimiento a sus educandos [7].

En este contexto, la presente investigación es de suma importancia en el ámbito educativo puesto que coadyuva al mejoramiento del proceso de enseñanza-aprendizaje que se imparte en la educación primaria de la ciudad de Esmeraldas al incluir la metodología STEAM para estimular el desarrollo del conocimiento y las destrezas de los educandos en los establecimientos educativos de la localidad. El tema de investigación forma parte del proyecto Implementación de la metodología STEAM en la educación básica y media en la ciudad de Esmeraldas, el proyecto cuenta con la aceptación en el Congreso Internacional de Investigación en Educación - CIINED - 2021 con la ponencia titulada Análisis de la Robótica Educativa en los primeros años de educación básica de la ciudad de Esmeraldas. 
De igual manera se cuenta con el artículo publicado por [8], titulado Implementación de un robot móvil con punto de acceso basado en la metodología STEAM.

Con el desarrollo de la presente investigación se pretende dar respuesta a la interrogante ¿Las aplicaciones basadas en la metodología STEAM contribuyen a mejorar los procesos de enseñanza-aprendizaje de la educación básica de la ciudad de Esmeraldas? Para poder dar respuesta a la hipótesis planteada, se desarrolló un juego interactivo basado en la metodología STEAM. La interfaz cuenta con una interfaz didáctica que le permitirá al educando de manera sencilla y interactiva el desarrolle de sus inteligencias múltiples enseñando desde tempranas edades los fundamentos de programación. La interfaz desarrollada antes de ejecutar el proceso emula que las instrucciones ingresadas sean las adecuadas para cumplir el reto, una vez verificado que no haya errores el software simula el funcionamiento de las instrucciones que son envidas por Wi-Fi al robot desarrollado por [8] para su funcionamiento en el mundo real.

El artículo se encuentra estructurado de la siguiente manera: en la Sección 2 se presenta un breve estado del arte, la metodología empleada se da a conocer en la Sección 3. Los resultados alcanzados se detallan en la Sección 4, mientras que; en la Sección 5 se realiza la discusión. En la Sección 6 se presentan algunas conclusiones fruto de la investigación realizada y finalmente, en la Sección 7 se da a conocer el trabajo a futuro.

\section{Estado del Arte}

\section{Competencia científica}

Para [9], la competencia científica es un constructo complejo que está integrado por tres categorías: conceptual, procedimental y epistémica. La capacidad conceptual, se consolida cuando el educando emplea patrones científicos para la argumentación. La capacidad procedimental, concretiza el uso de habilidades investigativas en las experiencias de aprendizaje. La dimensión epistémica, por su parte permite la validación del conocimiento basado en evidencias y argumentación en la que se cohesiona, el saber, el contexto, la controversia y el discurso científico.

\section{Enfoque transdisciplinario}

En [10], se indica que la transdisciplinariedad tiene tres características: a) la apertura a lo desconocido, b) el rigor mediante el proceso de indagación científica y la condición ética y c) la lógica de asumir el desafío en el mundo actual.

\section{Metodología STEAM}

La metodología STEAM es el acrónimo de la expresión Ciencia, Tecnología, Ingeniería, Arte y Matemática que junto a la robótica educativa, en la actulaidad asumen un rol importante en el ámbito educativo. La educación basada en la metodología STEM, le permite al educando tener una participación activa, aprender mientras juega y a su vez desarrolla el pensamiento crítico, el trabajo en equipo y la comunicación [11]. En la figura 1, se presenta las bases de la metodología STEAM. 


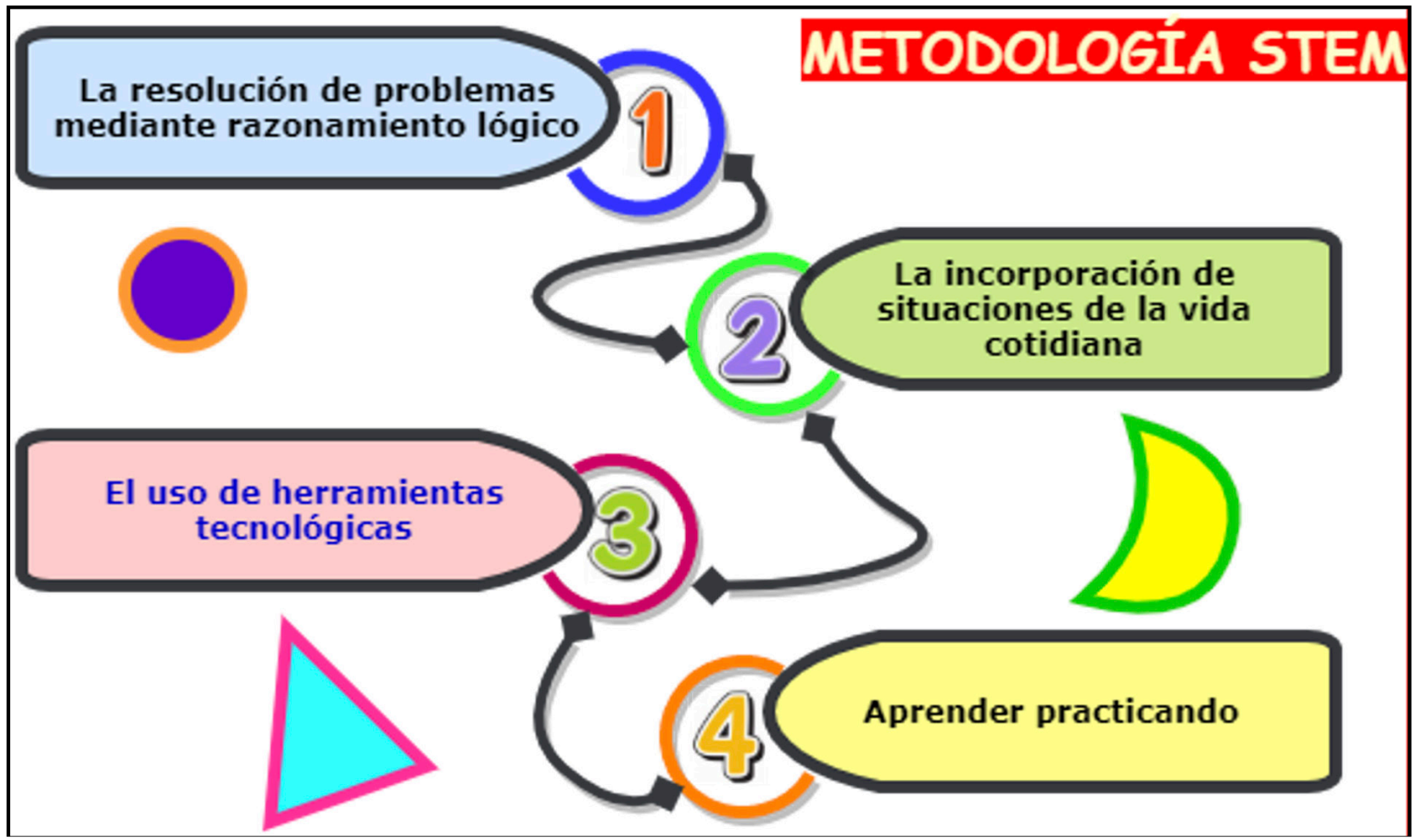

Fig. 1. Bases de la metodología STEAM.

Metodología STEAM basada en el Modelo TPACK. Según [12] es un modelo integralsistémico planteado por Koehler en el 2012. TPACK es el acrónimo de la expresión Tecnología, Pedagogía y Contenido, este modelo integra los conocimientos tecnológicos que integra las TIC, de contenido y el pedagógico relacionado a las estrategias de aprendizaje.

Aprendizaje basado en la Robótica Educativa. En [13], manifiestan que la robótica educativa es propicia para apoyar habilidades productivas, creativas, digitales y comunicativas generando cambios aptitudinales y actitudinales positivos tanto en educadores como en educandos.

\section{Metodología}

Se inició la investigación realizando una revisión sistemática empleando el método PRISMA, la cual consiste en aplicar un conjunto de ítems para la selección y el análisis de los artículos a analizar. Se emplearon como criterios de inclusión y exclusión: a) el año de publicación: tomando en cuenta los artículos científicos publicados en los últimos 5 años, b) idioma de publicación: se consideran artículos tanto en inglés como en español y; c) tipología de estudio: se toman en cuenta estudios que aborden procesos curriculares basados en la metodología STEAM en cualquier tipo de investigación.

La búsqueda sistemática se llevó a cabo en las bases de datos SCOPUS, Dialnet, ScienceDirect y Google Académico, utilizando como palabras clave en inglés "STEAM education", "STEAM Methodology" and "STEAM Aplications" y en español "educación STEAM", "metodología STEAM" y "Aplicación STEAM". Para mejorar el sesgo de la búsqueda se empleó el sistema booleano AND delimitando la búsqueda al título, el resumen y las palabras clave. De los artículos seleccionados se elaboró una base de datos tomando en cuenta autor, año, título, palabras clave, relación con el estudio y resultados; esta base se exportó al software Mendeley por medio del cual se eliminaron los documentos duplicados. 
Finalmente, se procedió a realizar una lectura crítica a texto completo de los documentos en función de los objetivos a fin de establecer la validez y calidad metodológica de los estudios seleccionados.

\section{Modelo de desarrollo en Espiral}

Para la creación de la aplicación del juego interactivo basado en la metodología STEAM, se utilizó el modelo de desarrollo evolutivo en espiral propuesto por Barry Boehm en 1986. Al aplicar el modelo en espiral, el software alcanza la madurez requerida gracias a la serie de entregas evolutivas proporcionadas en cada ciclo repetitivo [14]. En la figura 2, se presenta el esquema del modelo en espiral empleado.

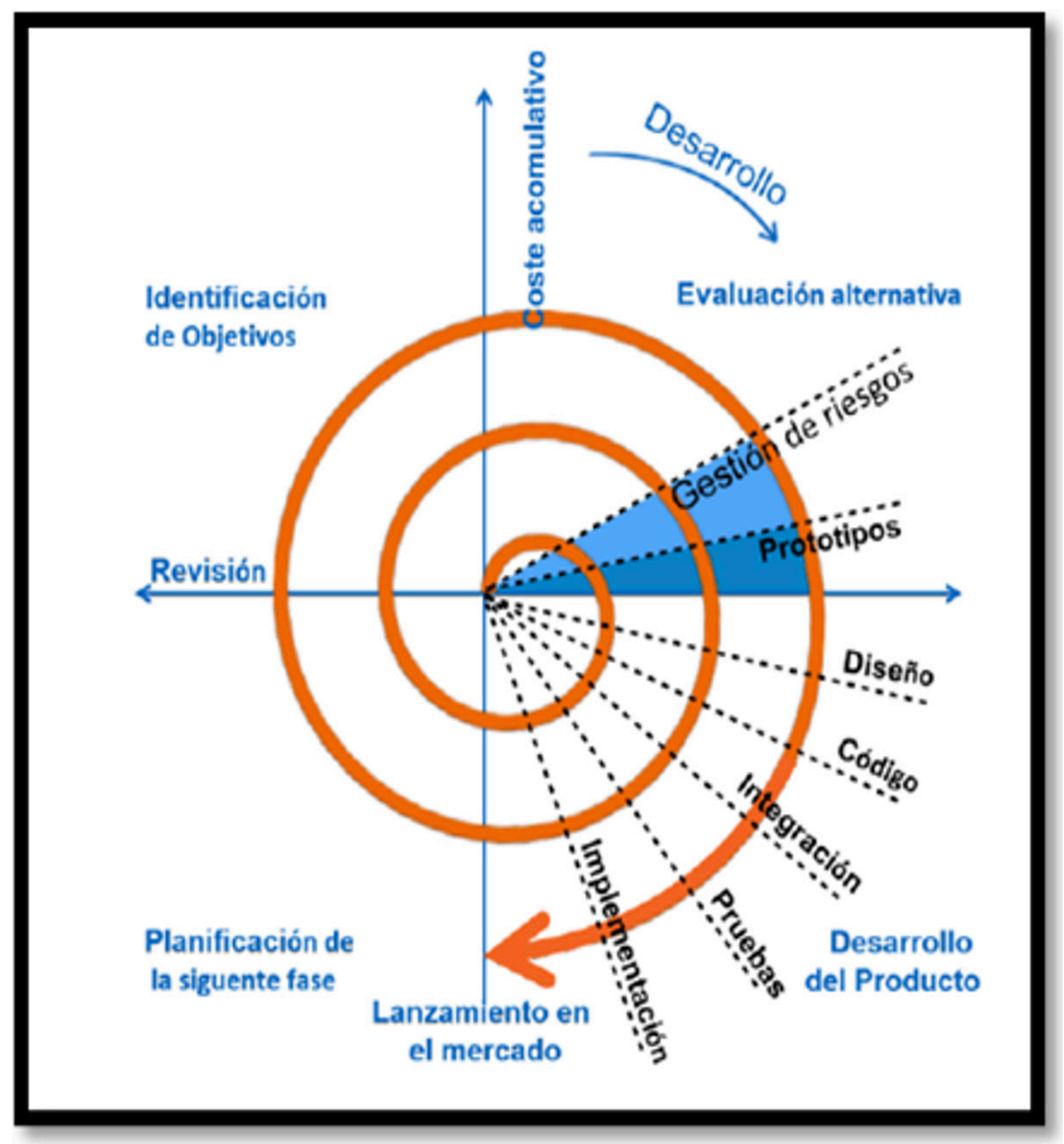

Fig. 2. Modelo de desarrollo evolutivo (espiral).

Fuente: Recuperado de [14].

\section{Diagrama de flujo de la aplicación}

El diagrama de flujo de la figura 3, representa la lógica con la que fue desarrollada el juego interactivo y a la vez muestra la secuencia de actividades que se deben realizar para una correcta interacción entre el usuario y la aplicación. 


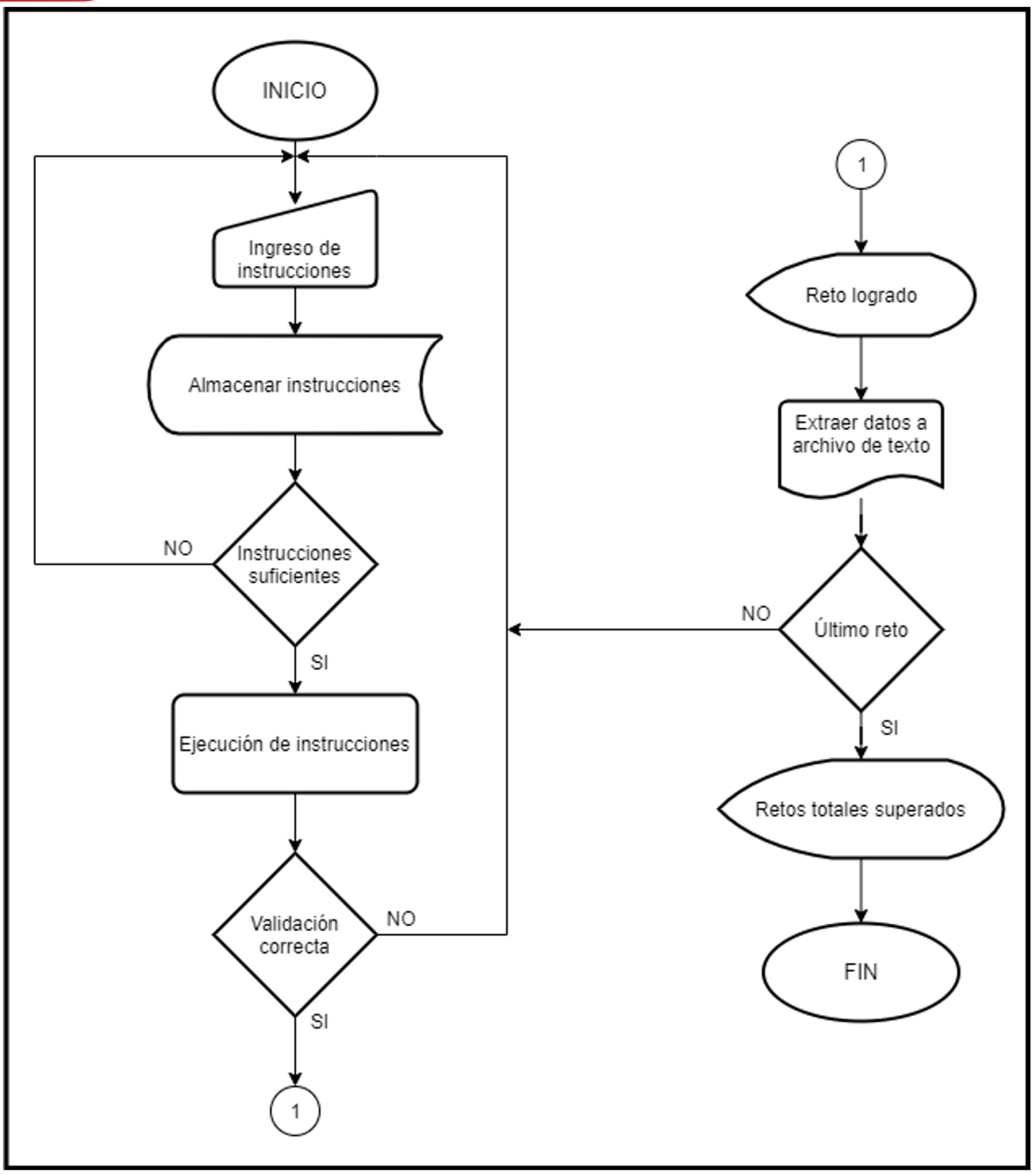

Fig. 3. Diagrama de flujo de la interactividad usuario-aplicación.

\section{Diseño de la interfaz}

El diseño de la interfaz se realizó utilizando los objetos predefinidos del lenguaje de programación Visual Basic, los mismos cuentan con propiedades ya establecidas que facilitan el desarrollo de la aplicación. En la tabla 1, se muestran el conjunto de objetos utilizados en el desarrollo del juego interactivo con su respectivo gráfico y nombre. La interfaz desarrollada es llamativa, amigable e interactiva para que los usuarios la puedan utilizar de forma intuitiva. 
Tabla 1. Objetos de la interfaz.

\begin{tabular}{lcl}
\hline \multicolumn{1}{c}{ Objeto } & Gráfico & \multicolumn{1}{c}{ Nombre } \\
\hline Botón de comando & - & Btn_iniciar \\
Caja de texto & Txt_enviar \\
Etiquetas & A & Lbl_título \\
Figuras & Sha_reto \\
Imagen & Img_herr \\
Cronómetro & Tim_reto \\
Marco & Fra_proceso \\
\hline
\end{tabular}

\section{$4 \quad$ Resultados}

El código fuente del juego interactivo basado en la metodología STEAM y un video explicativo se encuentran subidos en el repositorio de GitHub disponibles en el siguiente link: https://github.com/jwsq1982/aplicaci-n-tortuguita-stem.git

La interfaz está compuesta por tres espacios bien diferenciados: a) panel de herramientas, b) área de procedimiento y c) área de juego. Las tres áreas anteriormente mencionadas, son el resultado de la combinación de los objetos descritos en la sección anterior y se explica en los siguientes apartados cada una de las mismas.

\section{Panel de herramientas}

Este panel está constituido por las instrucciones que el educando debe utilizar para cumplir los retos que se presentan en el área del juego. Con la finalidad de llamar la atención de los niños y que puedan diferenciar fácilmente las instrucciones, se emplearon figuras geométricas de distintos colores. En la figura 4, se presentan las figuras geométricas con las instrucciones que ejecutan en el juego.

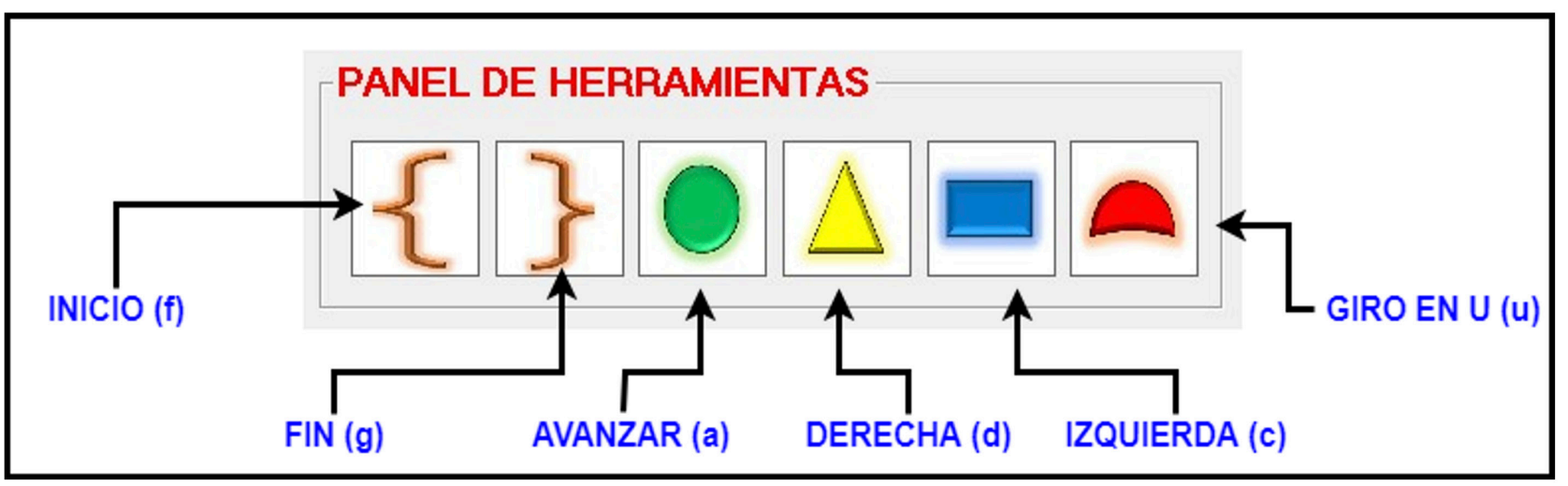

Fig. 4. Panel de herramientas del juego interactivo. 
Las instrucciones deben ser arrastras desde el panel de herramientas hasta el área de procedimiento, al realizar esta actividad los niños están aprendiendo figuras geométricas, colores, razonamiento lógico y matemático, ubicación espacial y motricidad de una forma lúdica.

\section{Área de Procedimiento}

Esta área es una matriz en la que se pueden colocar un máximo de 16 instrucciones como se puede observar en la figura 5. Con la finalidad de enseñar indirectamente los principios básicos de programación, los niños deben colocar el set de instrucciones dentro de las llaves para que el botón Ejecutar se habilite. Al presionar el botón Ejecutar, el set de instrucciones es simulado en el área de juego con el movimiento animado de la tortuga.

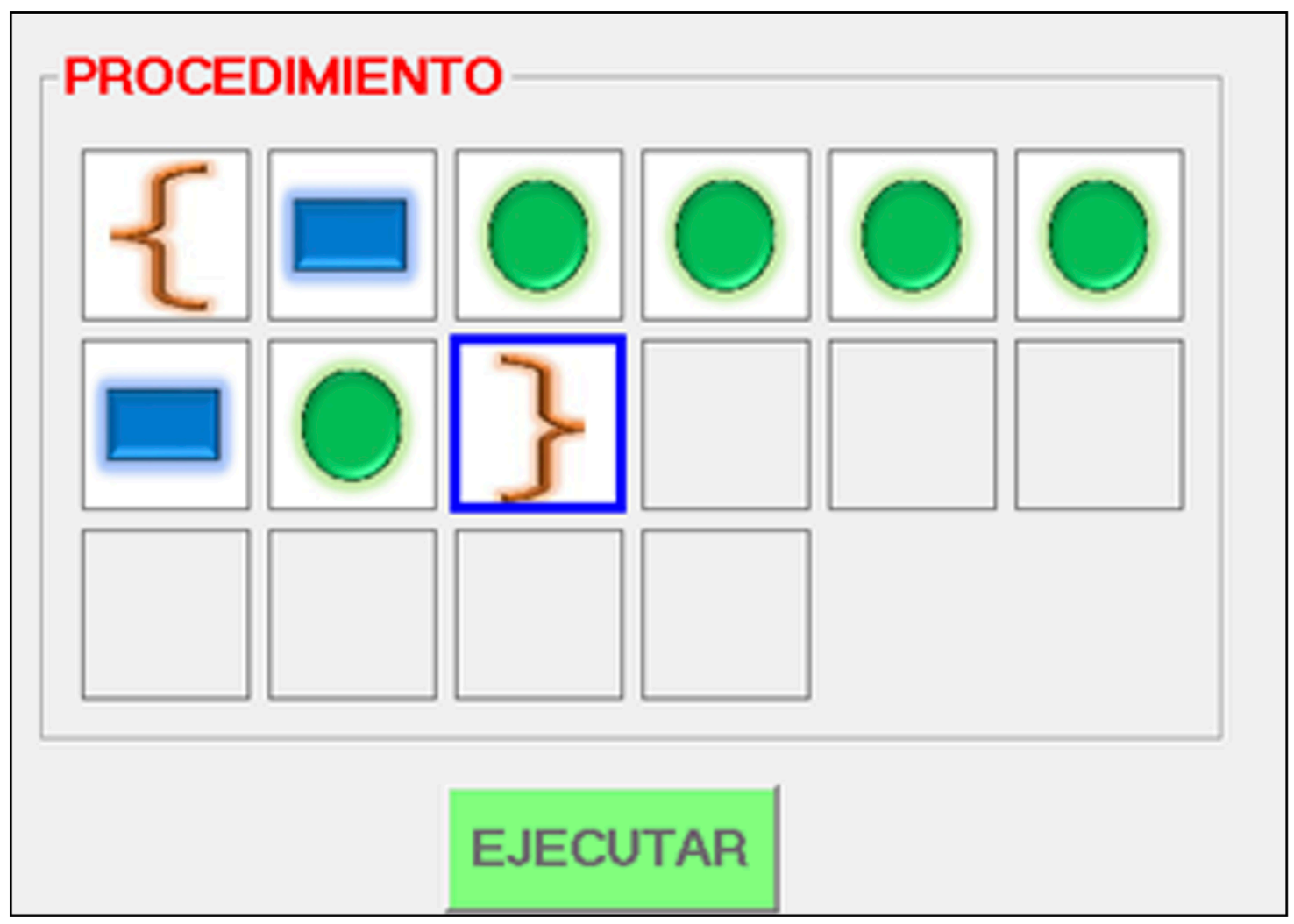

Fig. 5. Panel de procedimiento del juego interactivo.

\section{Área de juego}

En esta área se presenta los retos que el educando debe superar, el camino es estático pero el fondo puede ser cambiada por el usuario desde el menú Configuración. En la figura 6, se presenta el área de juego con la tortuga y los retos planteados; por defecto todos los retos estarán de color gris y conforme vaya avanzando los mismo se irán coloreando.

En esta área el niño podrá ver que la tortuga realiza cada una de las instrucciones que se colocaron en el panel de procedimiento de forma animada. Si el educando consigue superar un reto, se muestra un mensaje como el de la figura 7; a la vez aparece un botón en el área de procedimiento denominado Enviar datos como se puede ver en la figura 8. 


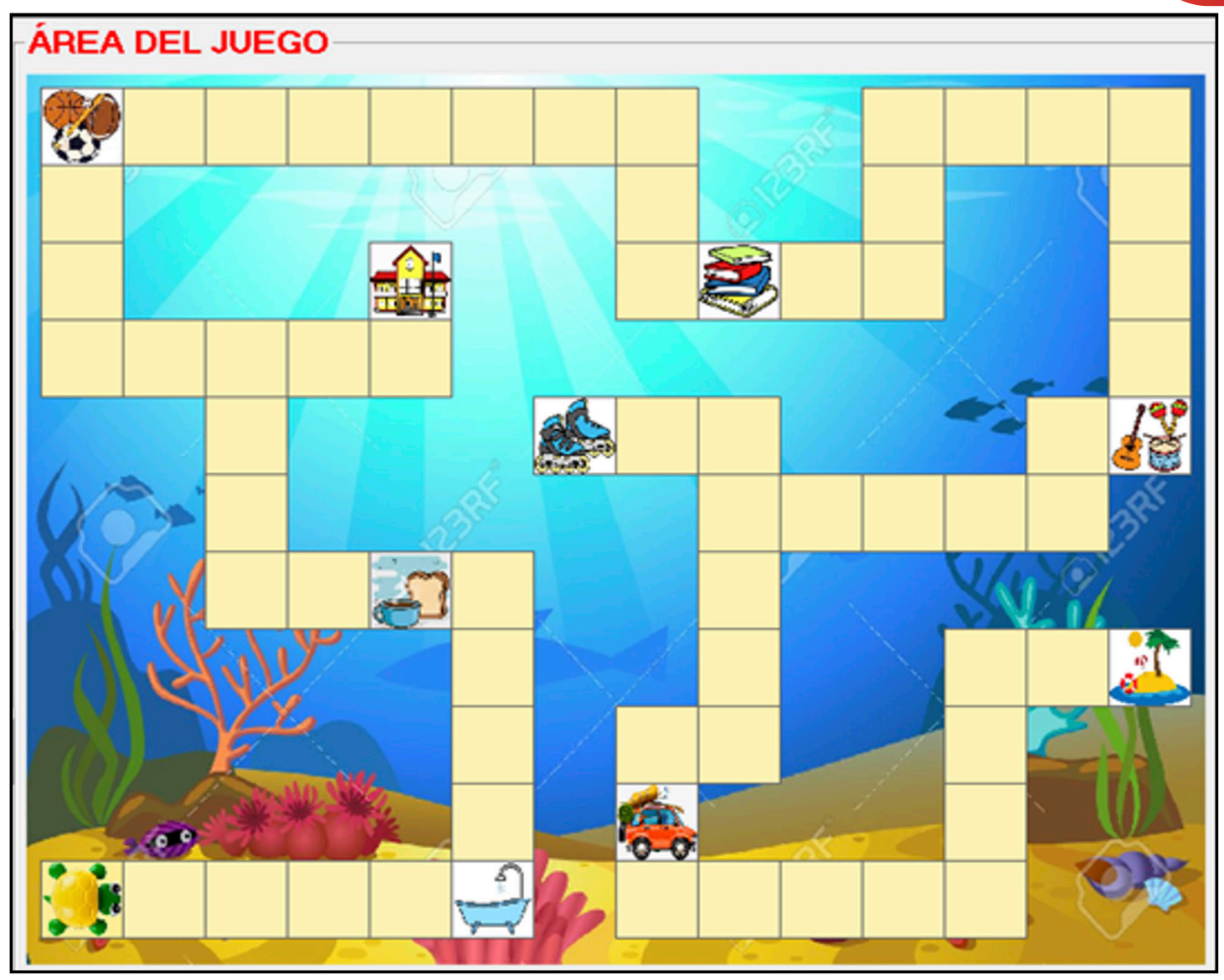

Fig. 6. Área de juego.

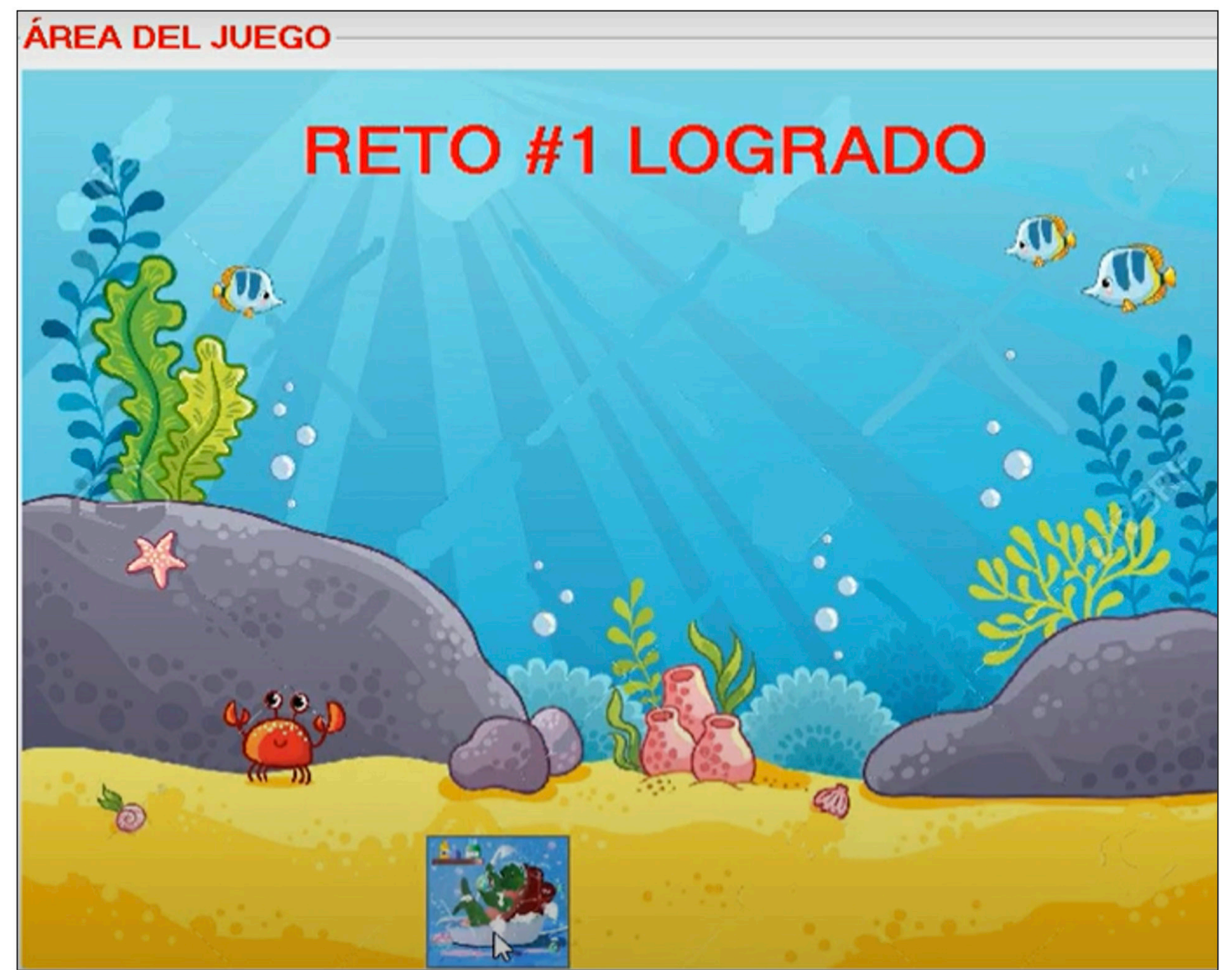

Fig. 7. Mensaje mostrado al superar un reto. 


\section{PAPER OG}

\section{PROCEDIMIENTO}
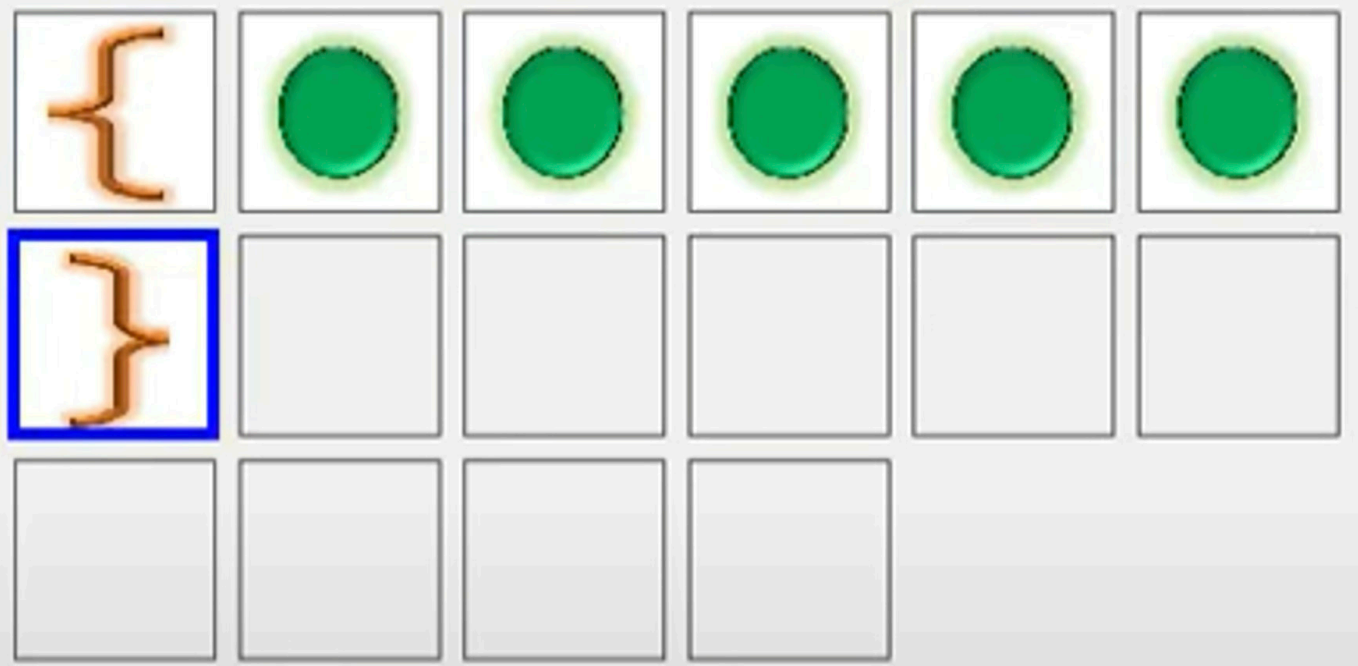

\section{EJECUTAR}

\section{ENVIAR DATOS}

Fig. 8. Botón para enviar instrucciones por Wi-Fi.

Este botón se habilitará cada vez que el niño supere el reto, con el cual se envían las instrucciones por Wi-Fi al robot móvil desarrollado por [8] y mostrado en la figura 9. El robot móvil se desplaza sobre una lona que contiene los mismos retos y forma que el área de juego digital, con esto se consiguió el objetivo de la metodología STEAM de vincular el software con e hardware y que el educando aprenda y desarrolle habilidades y destrezas mientras juega.

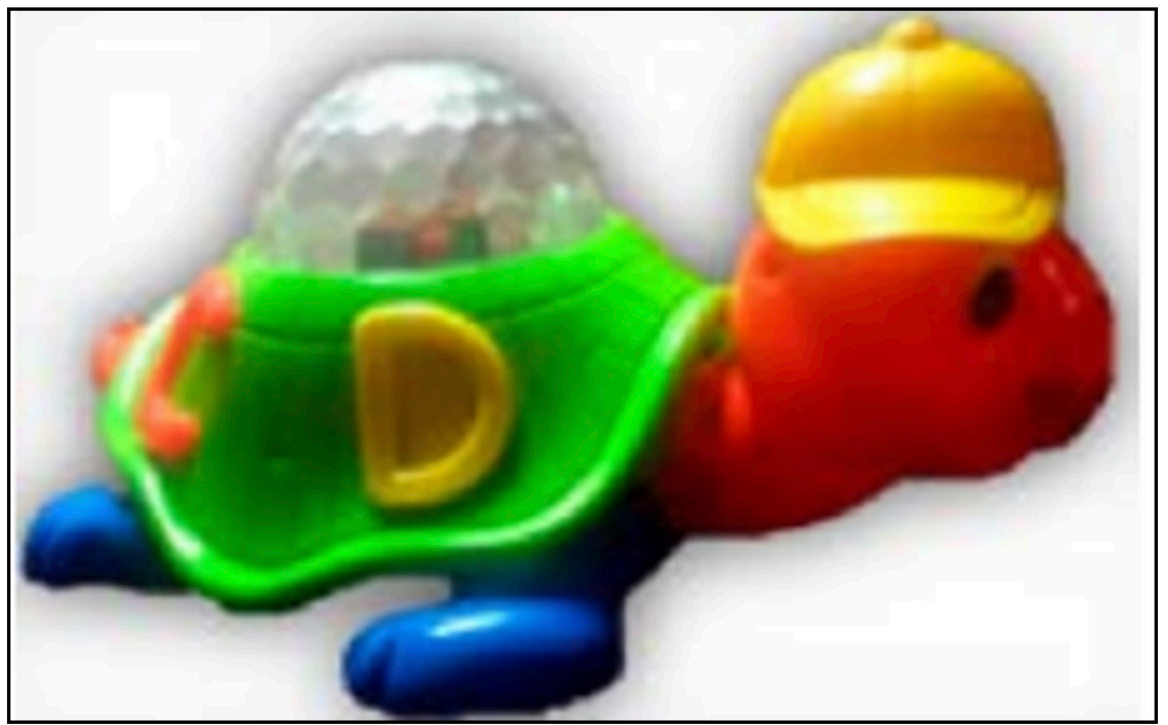

Fig. 9. Robot móvil.

Fuente: Disponible en [8]. 


\section{Discusión}

En la investigación bibliográfica realizada no se encontraron aplicaciones similares a la planteada, la mayor parte de investigaciones como las realizadas por [4], [6], [7], [11], [13] se basan únicamente en fundamentos teóricos o en aplicaciones web que no interactúan con los niños en el mundo real.

El juego interactivo implementado basado en la metodología STEAM contribuye al desarrollo integral de los niños al mejorar destrezas psicomotrices (arrastrar y soltar), el razonamiento lógico (direcciones), razonamiento matemático (conteo), habilidades cognitivas (figuras y colores) y el razonamiento espacial. Cabe mencionar que este proyecto es parte de un programa de investigación con el cual se está buscando integrar la metodología STEAM desde los primeros años de educación básica creando hardware y software propios de bajo costo.

Finalmente, con el juego interactivo implementado se consiguió dar respuesta a la hipótesis planteada y contribuir con la misma en el desarrollo integral de los educandos al emplear la tecnología en el proceso de enseñanza-aprendizaje.

\section{Conclusiones}

- El juego interactivo desarrollado basado en la metodología STEAM es novedoso, interactivo e intuitivo, ya que por medio de la tecnología permite a los educandos aprender mientras juegan.

- Con la aplicación implementada los niños desarrollan varias habilidades y destrezas simultáneamente como el razonamiento lógico, razonamiento matemático, ubicación espacial, motricidad y lógica de programación.

- Por medio de la simulación animada, el usuario puede verificar si las instrucciones colocadas en el área de procesamiento son las adecuadas para superar el reto.

- El área de juego tiene retos de actividades diarias como bañarse, comer, ir a la escuela, entre otras y puede cambiarse el fondo para de esta forma captar la atención de los niños; de igual manera se utilizó figuras geométricas y colores para que los educandos puedan relacionar las instrucciones de una u otra forma y la tortuga como medio animado por ser un icono representativo del Ecuador.

- Al vincular el juego interactivo con el robot móvil, debe verificarse que la distancia máxima entre ambos sea inferior a cinco metros para evitar problemas de conexión.

\section{Trabajo a futuro}

El juego interactivo es parte de un proyecto de investigación el cual al momento cuanta con los trabajos de investigación descritos en la introducción, razón por la cual; se está trabajando en la próxima fase del proyecto la cual consiste en implementar un tablero de programación físico el cual se vinculará con la aplicación. El objetivo es que el niño coloque las instrucciones en el tablero, estas sean enviadas al simulador y si no hay errores la aplicación envíe las instrucciones al robot móvil.

De igual manera, al momento el juego no incorpora ciclos repetitivos o de decisión por lo que se trabajará en añadir estas características para poder emplear el mismo hardware y software en niños de mayor edad. 
Finalmente, por medio de la Coordinación de Vinculación con la Sociedad y la carrera de Desarrollo Infantil Integral del ISTLT, se capacitará a los docentes de educación básica de la ciudad de Esmeraldas en el uso del juego y del robot móvil para medir el verdadero impacto que se puede alcanzar al emplear la metodología STEAM en los procesos de enseñanza-aprendizaje.

\section{Referencias}

E. G. Parés, "Aplicaciones web 3.0 para la mejora del aprendizaje," DIM Didáctica, Innovación y Multimed., vol. 0, no. 39 SE-Articles, Jun. 2021, [Online]. Available: https://raco.cat/index.php/DIM/article/view/388775.

F. R. Sheu and M. Shih, "Evaluating NTU's OpenCourseWare project with Google Analytics: User characteristics, course preferences, and usage patterns," Int. Rev. Res. Open Distance Learn., vol. 18, no. 4, pp. 100-122, 2017, doi: 10.19173/irrodl.v18i4.3025.

F. Rentería, E. Palma, and J. Castro, "Utilización de aplicaciones web para optimizar el proceso de enseñanza aprendizaje," Rev. Clake Educ., vol. 2, no. 3, pp. 3-18, 2021, [Online]. Available: http://revistaclakeeducation.com/ojs/index.php/Multidisciplinaria/article/ view/82.

K. Gricelda, S. Maria, M. Esther, and P. Gamero, "Metodología STEAM en el desarrollo de competencias científicas en la educación básica," pp. 0-2, 2021.

S. Miranda Fuenzalida and E. Treviño, "Uso de TERCE-UNESCO para informar la práctica educativa: factores que influyen en el aprendizaje en Ciencias en Chile y Paraguay," Rev. Iberoam. Educ., vol. 84, no. 1, pp. 135-156, 2020, doi: 10.35362/rie8414025.

C. Conradty and F. X. Bogner, "STEAM teaching professional development works: effects on students' creativity and motivation," Smart Learn. Environ., vol. 7, no. 1, 2020, doi: 10.1186/s40561-020-00132-9.

M. Nevárez Toledo, "La robótica educativa como herramienta de aprendizaje colaborativo en estudiantes de educación general básica superior," p. 92, 2016, [Online]. Available: https://repositorio.pucese.edu.ec/handle/123456789/625.

F. Yépez, «IMPLEMENTACIÓN DE UN ROBOT MÓVIL CON PUNTO DE ACCESO BASADO EN LA METODOLOGÍA STEAM», I+D+i ISTCT, vol. 2, n.ํ 1, p. 10, jun. 2020.

F. I. De Prada Pérez De Azpeitia, "Infrared thermography: An amazing resource for teaching physics and chemistry," Rev. Eureka, vol. 13, no. 3, pp. 617-627, 2016, doi: 10.25267/ Rev.

Y. Castañeda Lozano, "La comunidad escolar: colectividad hacia la investigación transdisciplinar," Rev. Educ. y Ciudad, no. 38, 2020, doi: 10.36737/01230425. n38.2020.2311.

C. Pisco, S. Magdalena, C. V. Tania, H. C. José, and D. J. Diógenes, "El modelo STEM como recurso metodológico didáctico para construir el conocimiento científico critico de estudiantes de Física," Uma revisão da Lit. em publicações 2010 a 2016 sobre o ensino conceitos Fundam. Mecânica Quântica, vol. 12, no. 2, p. 6, 2018. 
B. L. COLORADO-AGUILAR and B. MORALES-GONZALEZ, "Avaliação dos conhecimentos tecnológicos, pedagógicos e disciplinares no ensino da ciência," Rev. IberoAmericana Estud. em Educ., vol. 13, no. 3, pp. 997-1010, 2018, doi: 10.21723/riaee. v13.n3.2018.11167.

I. Moreno, L. Muñoz, J. Serracín, J. Quintero, K. Pitti-Patiño, and J. Quiel, "La Robótica Educativa, una herramienta para la enseñanza-aprendizaje de las ciencias y las tecnologías.," Teoría la Educ. Educ. y Cult. en la Soc. la Inf., vol. 3, no. 2, pp. 74-90, 2012.

C. Fuchs and K. Grützner, "Digitales," Praxishandb. Kongress-, Tagungs- und Konf., pp. 447-455, 2017, doi: 10.1007/978-3-658-08309-0_29. 


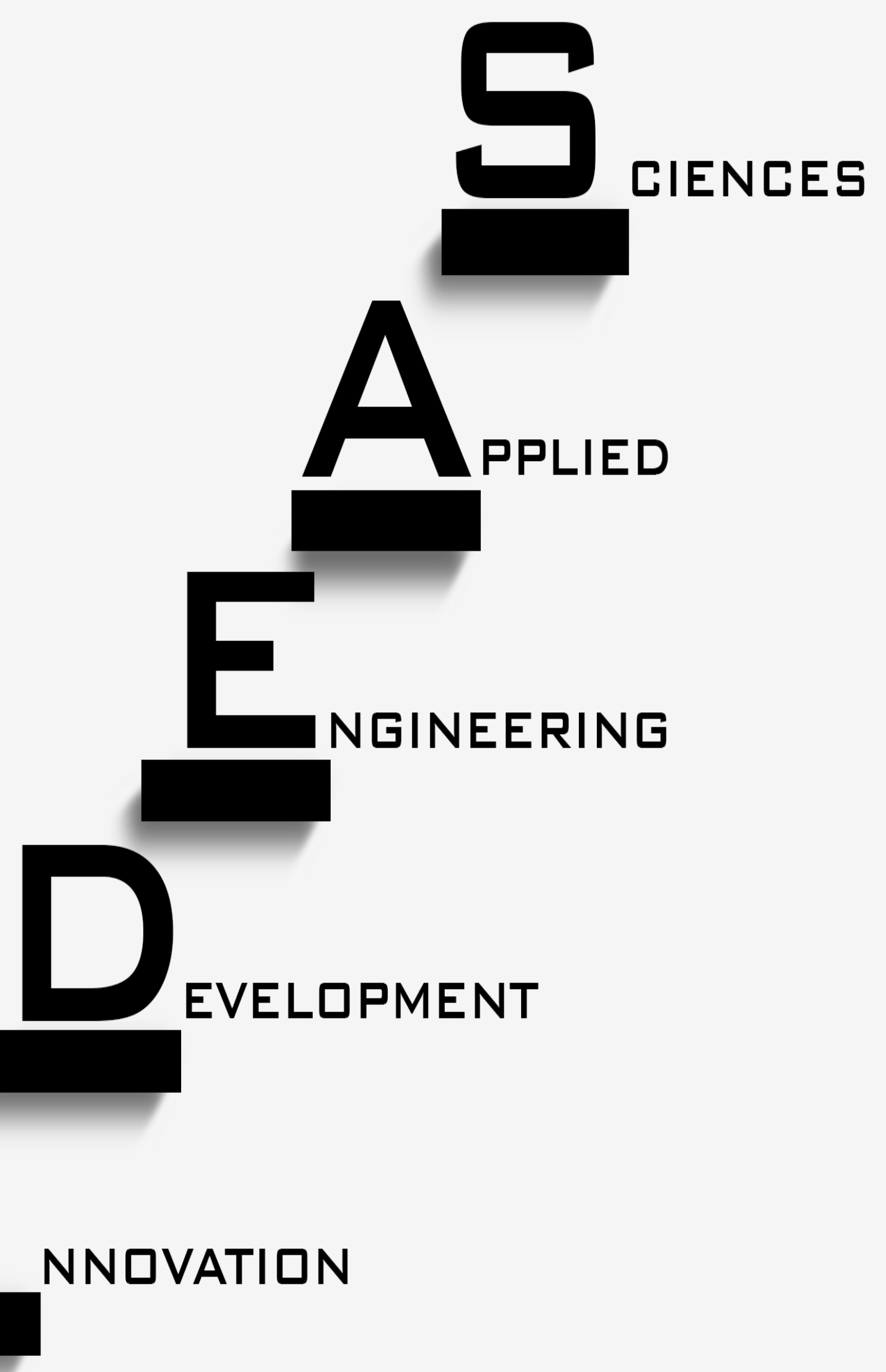





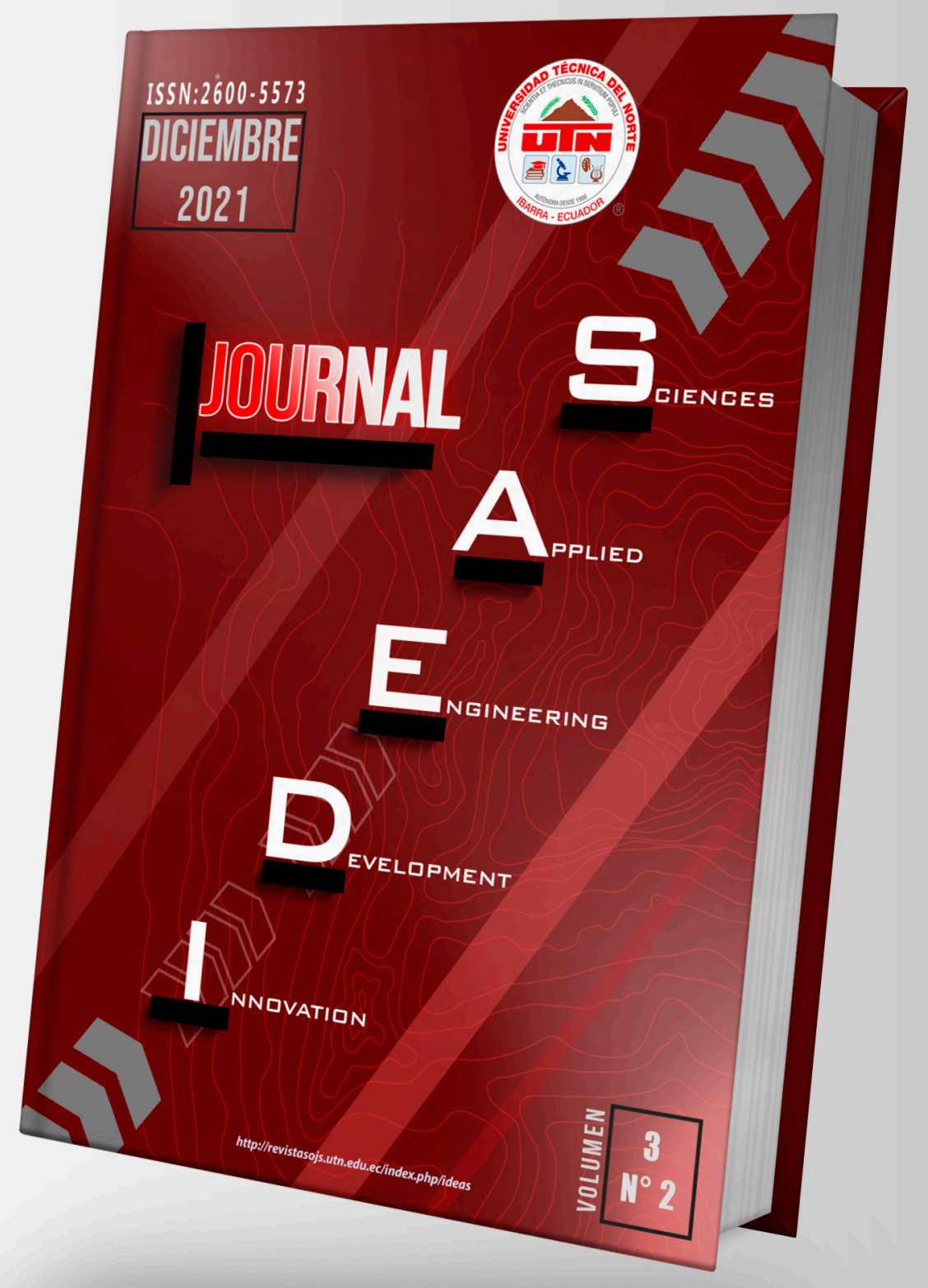

